\title{
Possession type affects resolution of possessive pronouns in English VP ellipsis
}

\author{
Jesse Storbeck \& Elsi Kaiser*
}

\begin{abstract}
The sentence "Bill washed his car, and John did, too" has two possible interpretations if the overt his refers to Bill: (i) a coreferential interpretation, in which John washed Bill's car, or (ii) a bound variable interpretation, in which John washed his own car. What guides comprehenders' selection of one over the other? Previous research has identified factors such as processing economy (e.g. Reuland, 2001) and lexical semantic properties of the verb and possessed noun (e.g. Foley et al., 2003; Ong \& Brasoveanu, 2014). We extend research on the contribution of possession type to resolution of this type of ambiguous VP ellipsis. We hypothesize that the range of possession types found in natural language varies in the extent to which the possessum is processed as an independent discourse referent or as dependent on the discourse representation of its possessor. Moreover, we expect that such differences modulate the possessum's availability for coreference and, therefore, affect ambiguity resolution. We conducted an experiment testing how different possession relations modulate adult L1 English speakers' interpretational preference. Inanimate nouns favored bound variable interpretations more than animates did, supporting our hypothesis that the overt possession's animacy and its resultant discourse status are important factors in the resolution of the elided possessive pronoun. Follow-up experiments confirmed these results and ruled out nouns' real-world plausibility of possession as a determinant of interpretational preference. Our results suggest that animate possessions are more likely than inanimates to receive independent status in the discourse and consequently to be available for coreference when the ellipsis is interpreted.
\end{abstract}

Keywords. sentence processing; ambiguity resolution; variable binding; coreference; discourse; possessives; VP ellipsis

1. Introduction. Pronoun interpretation can occur via two mechanisms: discourse-level coreference or semantic-level binding (e.g. Heim, 1982; Reuland, 2001). These mechanisms yield different interpretations of the VP ellipsis in a sentence like Example (1a).

(1) a. Bill washed his car, and John did, too.

b. Bill $\mathrm{i}_{\mathrm{w}}$ washed his $\mathrm{i}$ car, and $\mathrm{John}_{\mathrm{j}}$ did [vp washed his $\mathrm{s}_{\mathrm{j}}$ car], too.

Bill [ $\lambda x$ ( $x$ washed $x$ 's car ) ], and John [ $\lambda x$ ( $x$ washed $x$ 's car ) ], too.

c. Bill i washed his $_{\mathrm{i}}$ car, and $\mathrm{John}_{\mathrm{j}}$ did [vP washed his $\mathrm{c}$ car], too.

Bill [ $\lambda \mathrm{x}(\mathrm{x}$ washed hisBill car $)]$, and John $[\lambda \mathrm{x}(\mathrm{x}$ washed hisBill car $)]$, too.

d. Bill ${ }_{i}$ washed hisk car, and $\mathrm{John}_{\mathrm{j}}$ did [vP washed his $\mathrm{s}_{\mathrm{k}}$ car], too.

Bill [ $\lambda \mathrm{x}(\mathrm{x}$ washed hisTed car $)]$, and John [ $\lambda \mathrm{x}(\mathrm{x}$ washed hisTed car $)]$, too.

Example (1b) is an instance of variable binding, in which his represents Bill in the first clause but John in the second ("sloppy identity"). Examples (1c) and (1d), on the other hand, are instances of coreference, in which the possessive pronoun his has the same reference - Bill or a

\footnotetext{
* The authors gratefully acknowledge helpful feedback from members of the USC Language Processing Lab and the audience at the 2017 California Meeting on Psycholinguistics. Authors: Jesse Storbeck, University of Southern California (jstorbec@usc.edu) \& Elsi Kaiser, University of Southern California (emkaiser@usc.edu).
} 
discourse-relevant third party - in the antecedent VP as it does in the elided constituent ("strict identity"). The interpretation in (1d) necessitates the presence of a salient third party in the discourse, and, without such a referent available, this interpretation is unlikely. It is reasonable also to expect that other situation-specific knowledge will influence interpretation of (1a). For example, the knowledge that John does not own a car would likely bias a comprehender against the bound variable interpretation in (1b); nevertheless, (1b) and (1c) are grammatical interpretations of (1a) without any supporting context. Given this ambiguity, what guides comprehenders' choice between coreferential and bound variable interpretations of VP ellipsis?

1.1. BASELINE BOUND VARIABLE BIAS. There is a converging body of research that variable binding is generally preferred over coreference in VP ellipsis. While online studies of comprehension are somewhat less concordant in this regard (e.g. Frazier \& Clifton, 2000; Shapiro \& Hestvik, 1995; Shapiro et al., 2003), there is clear evidence demonstrating that in offline comprehension by typical adult populations, bound variable interpretations of VP ellipsis are privileged relative to coreference (e.g. Fiengo \& May, 1994; Koorneef et al., 2011). Reuland (2001) has proposed that this preference emerges because variable binding is the more economical interpretation; the Primitives of Binding framework (Reuland, 2001) introduces an economy hierarchy of pronominal reference assignment, where operations taking place at the level of semantics (i.e. variable binding) are less costly than operations at the level of discourse (i.e. coreference).

Results from child language acquisition studies also support the claim that variable binding is a linguistic primitive - or is at least computationally simpler (e.g. Foley et al., 2003; Guo et al., 1996). Foley et al. found in both an act-out task and a truth value judgment task that all age groups tested $(3,0-7,11)$ had a bias towards bound variable interpretations. Additionally, while all age groups demonstrated at least some competence with coreferential interpretations, older children offered more coreferential interpretations in the act-out task and were more successful at endorsing coreference in truth value judgments. These results support the claim that coreferential interpretations are more subject to maturational constraints than variable binding is.

Studies of agrammatic patients complement the results from the L1 acquisition literature by demonstrating that Broca's aphasia significantly impairs the ability to compute coreferential interpretations but affects variable binding to a lesser degree (e.g. Grodzinsky et al., 1993; Vasić et al., 2006). In Vasić et al.'s study, patients with agrammatic Broca's aphasia matched pictures to target sentences containing Dutch VP ellipsis constructions. Patients performed above chance in matching depictions of bound variable interpretations to sentences but were at chance with coreferential interpretations. When patients could choose between two correct interpretations, they overwhelmingly chose the bound-variable option. Unimpaired controls also preferred bound variable interpretations, although to a lesser extent. Vasić et al. interpret their results according to the Primitives of Binding framework (Reuland, 2001): in unimpaired adults, the bound variable (semantic-level) interpretation of the antecedent VP becomes available earlier, followed shortly thereafter by the coreferential (discourse-level) interpretation; both interpretations are available by the time the parser resolves the ellipsis, and thus an ambiguity arises. In agrammatic patients, it is not the case that they are fully unable to compute coreference, but this operation's costlier nature means that the coreferential interpretation of the antecedent VP is not available in time to be copied to the ellipsis site.

Viewed holistically, prior research on the interpretation of ambiguous VP ellipsis suggests that -all other factors held constant - bound variable interpretations take precedence over coreferential ones. 
1.2. THE INFLUENCE OF LEXICAL SEMANTICS AND POSSESSION TYPE. Prior work suggests that semantic properties within the elided VP can influence interpretational preference. Ong \& Brasoveanu (2014) found that a verb's implicit causality modulated the strength of bound variable preference; specifically, they found in an offline interpretation task that subject-oriented implicit-causality verbs (e.g. aggravate, charm, frighten) produced more bound variable interpretations than object-oriented implicit-causality verbs (e.g. blame, envy, praise) across multiple discourse connective conditions. Additionally, Foley et al. (2003) found that self-directed verbs (e.g. scratch) resulted in more bound variable interpretations than other-directed verbs (e.g. move).

Lexical semantic properties of possessed nouns in sentences like Example (1a) have also been shown to modulate preference for variable binding. Foley et al. (2003) found that inalienable possessions (e.g. his arm) resulted in more bound variable interpretations than alienable possessions $^{1}$ (e.g. his apple). While Foley et al. suggest that the type of possession may influence a comprehender's choice between bound variable and coreferential interpretations, this work's applicability to adult sentence comprehension comes with a few caveats. Firstly, there is the straightforward limitation that the results they report from child learners may not accurately represent adult preferences. In addition, because of task constraints with this population, their set of nouns included only concrete, imageable concepts. Lastly, Foley et al. compared only inalienable and alienable possession relations. However, possessives also express relations such as kinship (e.g. her father) and other human associations (e.g. his boss). Haspelmath (2017) presents extensive evidence that in many languages, expressing different possession types requires different morphosyntactic mechanisms.

Maltese (Haspelmath, 2017)
a. id-i
hand-1sG.POSS
'my hand'
b. 'ktieb-i
book-1SG.POSS
'my book'
c. il-ktieb tiegh-i
ART-book, of-1SG
'my book'

Example (2) illustrates that the Maltese suffix which denotes inalienable/part-whole possession in (2a) is ungrammatical in a case of alienable/ownership possession (2b). Such cross-linguistic patterns support the theory that at least some possession types are cognitively distinct. Therefore, one might expect nominals expressing different possession types to be processed differently, even when they share morphosyntax (e.g. in English).

\footnotetext{
${ }^{1}$ Hollmann \& Siewierska (2007:410) offer the following definition of alienability based on semantic relations: "Inalienable possession is generally seen as involving a fairly stable relation over which possessors have little or no control, alienable possession as comprising a variety of less permanent, more controlled relationships." As Haspelmath (2017) notes, other definitions exist, and similar semantic relations may be more or less alienable crosslinguistically; nevertheless, this definition is sufficient for our purposes. Specifically, in our study, we adopt the standard view that body-part relations (e.g. her nose), abstract part-whole relations (e.g. his personality), and kinship relations (e.g. her mother) are examples of inalienable possession. We consider as alienable possession any relation expressing ownership of an item by a person (e.g. his bike, her newspaper).
} 
While Foley et al. (2003) and Guo et al. (1996) identify a possessed noun's alienability as a predictor of bound variable bias, previous work has also examined the influence of the possessum's animacy. Dahl \& Fraurud (1996:57) suggest that a difference in animacy affects the preferred interpretation of Examples (3a) and (3b) below.

(3) a. John sent his paycheck to his mother, and Bill sent it to his wife.

b. John sent his daughter to his mother, and Bill sent her to his wife.

They claim that (3a), in which the possessed noun paycheck is inanimate, is biased toward bound variable interpretation (i.e. that Bill sent his own paycheck to his wife); whereas (3b), in which the possessed noun daughter is animate and human, is biased toward coreferential interpretation (i.e. that Bill sent John's daughter to his wife). They speculate that these contrasting intuitions might "[have] to do with differences in individuation between animates and inanimates" (Dahl \& Fraurud, 1996:57). Nevertheless, the judgments they present are not supported by experimental evidence, nor do they quantify the extent to which animacy influences the relative bound variable biases of Examples (3a) and (3b). Our study addresses both of these research questions.

1.3. RESEARCH QUESTIONS AND AIMS. Although the relative ease of processing bound variable and coreferential interpretations is well-investigated, much less attention has been paid to how possession type modulates preference for variable binding. The experiments presented here aim to contribute to our understanding of how comprehenders interpret ellipsis construction like Example (1a), which are ambiguous between bound variable and coreferential construal. In this investigation, we focus specifically on the contribution of the possession relation and compare the relative influence of the possessed noun's animacy and alienability.

2. Experiment 1. We conducted an experiment testing how and whether different possession relations modulate preference for bound variable versus coreferential interpretations. The experiment examined four possession types: inalienable (e.g. nose), ownership (e.g. bicycle), animate relational (e.g. opponent), and kinship (e.g.father).

2.1. MethodS. The experiment was implemented in Qualtrics and used a simple two-alternative forced-choice task to probe participants' interpretational preference for ambiguous VP ellipsis constructions. Each item appeared on a separate page and required participants to confirm their selection by clicking an arrow to continue, as seen below in Figure 1.

Jason jolfed his bicycle, and Ronald did, too.

Ronald jolfed his own bicycle.

Ronald jolfed Jason's bicycle.

Figure 1. An example target item from Experiment 1 as it appeared to participants in Qualtrics 
Target items had the format in Example (4). Below each target sentence (4a), there were two answer choices. One represented the bound variable interpretation of the elided pronoun (4b), and the other represented the coreferential interpretation (4c). Participants were instructed to select the answer choice that they thought was most compatible with their interpretation of the sentence. The order of the bound variable and coreferential choices was counterbalanced across conditions.

(4) a. [Name 1] [nonce verb-PST] his/her [noun], and [Name 2] did, too.

e.g. Helen chabbed her jacket, and Amanda did, too.

b. [Name2] [nonce verb-PST] his/her own [noun].

e.g. Amanda chabbed her own jacket.

c. [Name2] [nonce verb-PST] [Name1]'s [noun].

e.g. Amanda chabbed Helen's jacket.

The key manipulation in the experiment was the possession type of the possessed noun. We tested four possession types: inalienable (e.g. nose, feelings, reputation), ownership (e.g. bicycle, jacket, newspaper), animate relational (e.g. opponent, colleague, roommate), and kinship (e.g. father, daughter, aunt). Inalienable nouns were all inanimate and consisted of a mixture of partwhole relations (i.e. body parts) and more abstract inalienables (e.g. reputation). Ownership nouns were also inanimate and denoted concrete entities typically understood to be the property of individuals. Animate relational nouns expressed a non-family relation between two people, whereas kinship nouns expressed a family relation. An example item in all four conditions is provided below as Example (5).

(5) Inalienable: Helen chabbed her nose, and Amanda did, too.

Ownership: Helen chabbed her jacket, and Amanda did, too.

Animate Relational: Helen chabbed her boss, and Amanda did, too.

Kinship: Helen chabbed her son, and Amanda did, too.

As in Examples (4) and (5), the names of the two "characters" in each target sentence were matched with respect to the gender most typically associated with them. This was done to prevent morphological mismatch between the overt and elided pronouns under bound variable interpretation ${ }^{2}$, e.g. Bill $i_{i}$ washed his $\boldsymbol{s}_{\boldsymbol{i}}$ car, and Mary did $_{\text {[VP }}$ washed her $\boldsymbol{r}_{\boldsymbol{j}}$ car], too. Nonce verbs (e.g. dreezed, jepped, swudged) were used in targets to minimize potential effects of verb semantics. This concern is a highly significant one in light of previous research demonstrating that verb type affects the availability of bound variable versus coreferential interpretations (Foley et al., 2003; Ong \& Brasoveanu, 2014). Another reason to prefer nonce verbs for this task was that the sentences should sound equivalently natural in all experimental conditions; however, many verbs which typically take human direct objects (e.g. chastise) sound odd with inanimates and vice versa.

The experiment contained 24 target and 40 filler items. Eight of the filler items were catch trials, where only one of the answer choices was possible, as in Example (6).

(6) Monica lives in a big city, and Whitney lives in the countryside.

[Choice 1] Monica lives in a rural location.

[Choice 2] Monica lives in an urban location.

\footnotetext{
${ }^{2}$ See $§ 5$ for a discussion of this type of gender mismatch.
} 
The other fillers contained ambiguities unrelated to ellipsis and pronoun resolution: prepositional phrase attachment ambiguities (e.g. Amber and Courtney meached the woman from far away: ambiguous between VP or NP attachment), scope/distributivity ambiguities (e.g. Cameron and Justin talked to a stranger at the rowdy bar: ambiguous relative position of existential quantification and conjunction), and lexical ambiguities (e.g. Steven and Samuel sprelled the injured fisherman's cast: ambiguous between multiple contextually-supported meanings of cast). Like target items, fillers always referred to two people with gender-matched names. Some fillers contained nonce verbs or nouns.

The experiment utilized a within-subject and within-item design, with the four possessiontype conditions distributed among experimental lists in a Latin square. Lists were pseudorandomized so that no target sentences occurred consecutively and the number of filler items between targets was variable. Four additional experimental lists were created by reversing the order of items in the lists generated by the Latin square, for a total of eight lists. Before beginning the experiment, participants saw five example items to familiarize them with the task.

2.2. PARTICIPANTS. Participants were recruited from Amazon Mechanical Turk. All participants were self-identified adult native English speakers who reported normal hearing and normal (or corrected-to-normal) vision. We excluded from analysis any participants who reported being born outside of the United States or scored below 7/8 correct on the catch trials. Here we report the results of 48 native speakers of American English with a mean catch trial score of 7.90/8.

2.3. PREDICTIONS. In line with previous research, we expect that the proportion of bound variable interpretations a sentence receives will vary as a function of the possession relation expressed by the noun in the antecedent VP. Additionally, we identify two competing hypotheses associated with prior work on the contribution of the possessed noun's alienability and animacy: (i) "the alienability hypothesis", in which the effect of alienability dominates, and (ii) "the animacy hypothesis", in which the effect of animacy dominates.

If the alienability hypothesis holds, then, following Haspelmath (2017), we expect that nouns in the inalienable condition will pattern with kinship nouns. These two conditions should also receive more bound variable interpretation than alienable ownership nouns, according to Foley et al. (2003) and Guo et al. (1996). The alienability hypothesis makes less specific predictions about the animate relational nouns, but it seems likely that they would pattern with the kinship nouns, since they express an abstract semantic relationship extremely similar to that of the kinship nouns.

On the other hand, if the animacy hypothesis holds, we expect that the inalienable and ownership nouns will pattern together, since they are both inanimate. According to Dahl \& Fraurud (1996), these inanimate nouns should receive more bound variable interpretations than animate relational and kinship nouns, which are all animate and human. 2.4 .

2.4. RESULTS. The results of Experiment 1 demonstrate that possession type influences the strength of bound variable bias $^{3}$ (see Figure 2). Inalienable and ownership nouns elicited equivalently high rates of BV interpretation $\left(77.5 \%\right.$ and $73.6 \%$ respectively; glmer $\left.{ }^{4}, p=0.24\right)$. The two inanimate conditions triggered more bound variable responses than animates $(47.6 \%$ for animate

\footnotetext{
${ }^{3}$ Note that for each condition, because of the binary nature of the task, the complementary percentage represents coreferential interpretations.

${ }^{4}$ Generalized linear mixed models for binomially distributed outcomes were used for all statistical comparisons. These analyses were performed using the lme4 package in R (R Core Team, 2016; Bates et al., 2015).
} 
relational and $31.3 \%$ for kinship nouns; $p<0.001$ ), reflecting an overarching effect of animacy. Further, relational nouns elicited more bound variable responses than kin terms $(p<0.001)$.

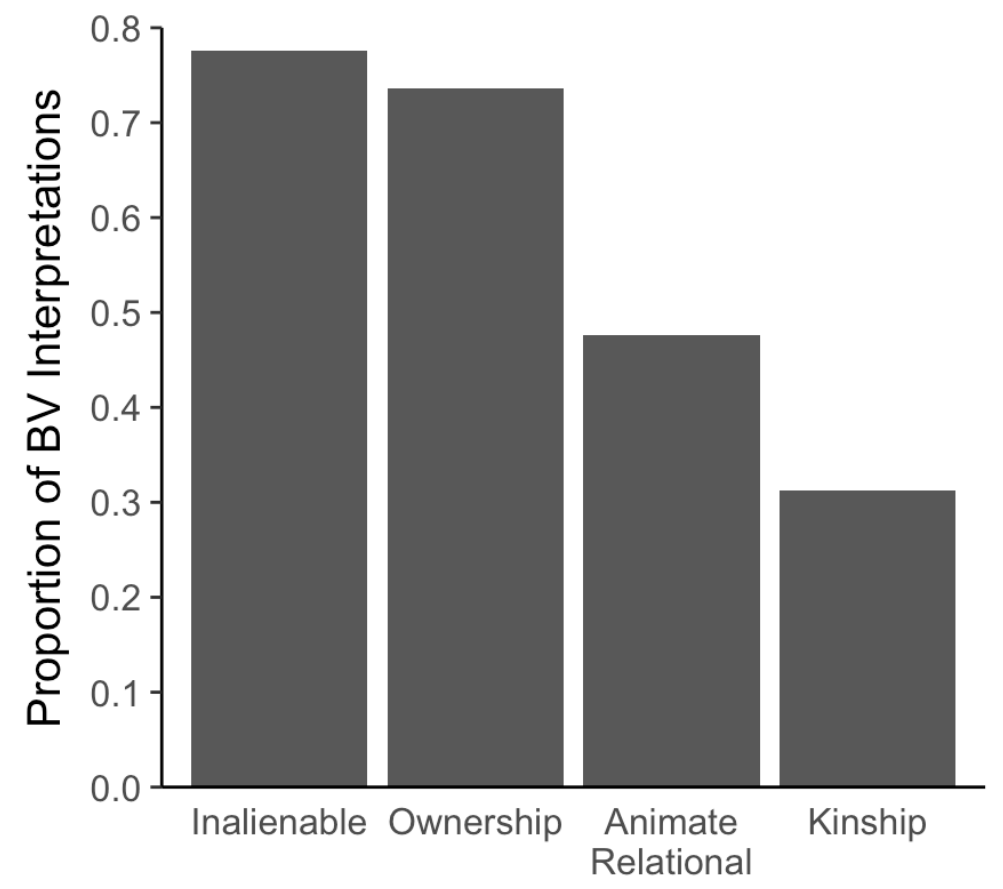

Figure 2. The proportion of bound variable responses by possession type in Experiment 1

2.5. DisCUSSION. Because the two inanimate conditions received high and similar rates of bound variable interpretation, and the inanimate conditions received fewer bound variable interpretations, the results of Experiment 1 support the animacy hypothesis in $\$ 2.3$. Our results contradict the predictions of the alienability hypothesis, since we find that inalienable and kinship nouns produce starkly different rates of bound variable interpretation, while the inalienable and ownership conditions do not differ. Therefore, we conclude that a possession's animacy is a stronger predictor of bound variable bias than its alienability.

To make sense of this pattern, we propose a simple model in the spirit of file change semantics (Heim, 1982). Firstly, we hypothesize that possessed nouns differ in the extent to which they are represented as independent discourse referents - as opposed to being dependent on the discourse representations of their possessors - and that animacy is one of a number of factors modulating this distinction. The model assumes that animate possessions are more likely to receive fully independent discourse representations, while inanimate possessions are less privileged and more likely to be conceptualized as dependent on the representations of their possessors. Secondly, this model posits that independent discourse entities are more easily available for coreference, in line with previous accounts that coreference requires access to discourse memory (Reuland, 2001). Consequently, the model assumes that dependent discourse entities resist coreference and, therefore, are more likely to receive bound variable interpretation, which Reuland (2001) argues operates at the level of semantics and without access to discourse memory.

At first blush, this type of model seems as if its operation is rather deterministic, with a given possessed noun always biased toward one interpretation or the other. This is not the finding of Experiment 1 and prior studies; therefore, it may be the case that an independent and dependent discourse representation can exist in parallel with differing levels of activation depending on a 
variety of factors; alternatively, the probabilistic patterns observed in Experiment 1 and prior research could fall out from variability in the dynamics of the representations themselves. In either case, this model's behavior is not deterministic but rather reflects a possessed noun's tendency to be processed as an independent or dependent discourse entity.

Accordingly, for Example (1a), "Bill washed his car, and John did, too", the competing discourse representations would be as shown in Figure 3.

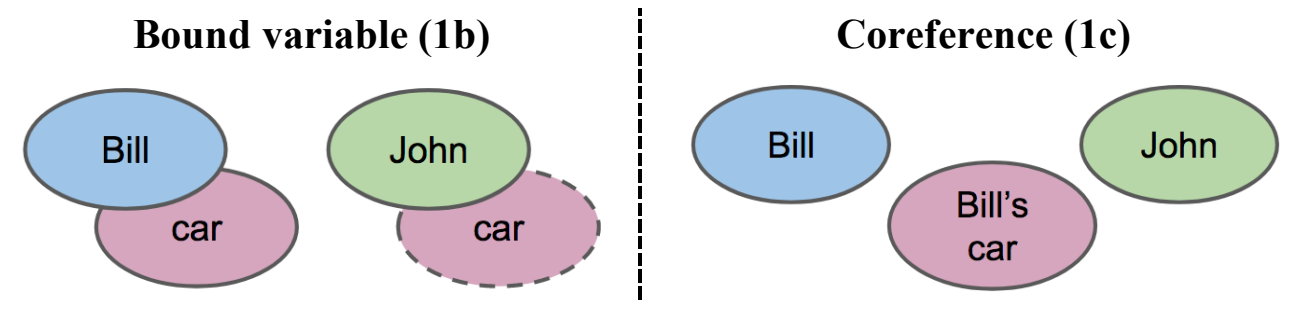

Figure 3. Bound variable and coreferential discourse schemata for Example (1a)

In this example, car is an inanimate possession; thus, when a comprehender processes "Bill washed his car...", car more readily receives a representation which depends on Bill. Given this representation, coreference is less likely when the ellipsis is interpreted because no independent representation for car is available. Instead, a second representation is built for car, this time dependent on John. Nevertheless, under the right circumstances, Example (1a) easily permits a coreferential interpretation. In these cases, we propose that the comprehender builds an independent representation for [Bill's] car, which is then available for coreference later when the ellipsis is interpreted. Again, the selection of representations is probabilistic and dependent on a variety of factors other than animacy; the core of our proposal is that animacy is a significant predictor of which representation will ultimately be selected.

A final point of discussion about the results of Experiment 1 is the surprising difference between the animate relational and kinship nouns. We expected these conditions not to differ, since kinship relations are a subset of the larger set of relationships which exist among people. However, the difference between these conditions seems robust (see \$2.6). In the framework of the model presented above, we attribute this difference to kin referents' high degree of cultural salience, which may lead to greater privilege and independence in their discourse representations. However, this topic deserves further investigation.

2.6. REPLICATIONS OF EXPERIMENT 1. Additional support for the pattern of results demonstrated in Experiment 1 comes from two additional studies that we conducted to address a rather different research question (see $\$ 5$ ). A full description of the motivations and results of these two studies is beyond the scope of this paper. Nevertheless, the methods and materials used for these studies were substantially similar to those of Experiment 1; all target items in these additional studies were constructed according to the same frame given in Example (4). These studies probed interpretation of sentences including ownership, animate relational, and kinship possession relations. Here we report the partial results of these studies (shown below in Figure 4) in comparison with the results of Experiment 1.

In cross-study comparisons, ownership nouns produced similarly high rates of bound variable interpretation in Experiment 1 and the replication studies (73.6\% and 80.2\% respectively, $\mathrm{p}=$ 0.22). Animate relational and kinship nouns produced even lower rates of bound variable interpretation in the replication studies relative to the results of Experiment $1(33.9 \%$ and $27.3 \%$ in replications; $47.6 \%$ and $31.3 \%$ in Experiment $1 ; \mathrm{p}<0.01$ ), further supporting the animacy hypothesis from $\$ 2.3$. As in Experiment 1, the animate relational and kinship conditions also 
differed significantly from each other $(\mathrm{p}<0.01)$, with animate relational nouns again producing the higher rate of bound variable interpretation.

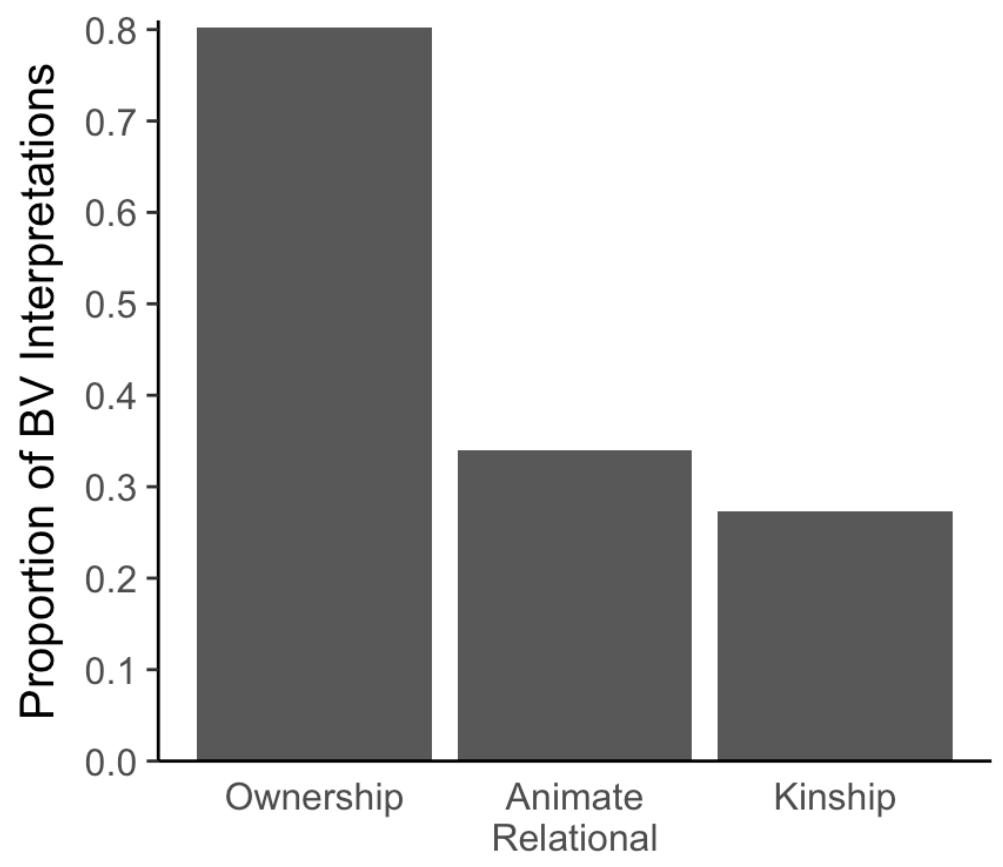

Figure 4. The proportion of bound variable responses by possession type in replication studies based on Experiment 1

3. Experiment 2. The results of Experiment 1 and replications thereof support our hypotheses about the roles of possession type and animacy in predicting bound variable bias for a given instance of VP ellipsis. Nevertheless, there could be an alternative explanation for the patterns we observe. Perhaps preference between bound variable and coreferential interpretations is determined simply based on real-world plausibility (e.g. in Example (1a), "Bill washed his car, and John did, too", the likelihood that John has his own car). For Example (1a), this theory would state that the more likely John is to have his own car, the more likely a comprehender is to interpret the ellipsis with variable binding; if John is unlikely to have his own car, coreference would be preferred. We conducted Experiment 2 in order to assess the validity of this alternative account.

3.1. MethodS. The experiment was implemented in Qualtrics. Participants were asked to rate "how likely an average person is to have at least one" of a series of nouns, including those used in Experiment 1. Ratings were on a 1-to-6 scale, with 1 signifying that "an average person is not likely at all to have at least one" and 6 signifying that "an average person is extremely likely to have at least one". Participants rated one noun at a time, as shown below in Figure 5. There were 65 total nouns tested in the experiment, which included all the possessed nouns which appeared in Experiment 1 and the replication studies. Each participant rated all 65 nouns. Some nouns which appeared as plural in Experiment 1 (e.g. shoes) were tested in their singular form in order to conform to the question format. Two pseudorandomized orderings of the items were created, and copies of each were reversed, yielding a total of four experimental lists. 
How likely is the average person to have at least one:

father

Not likely at all

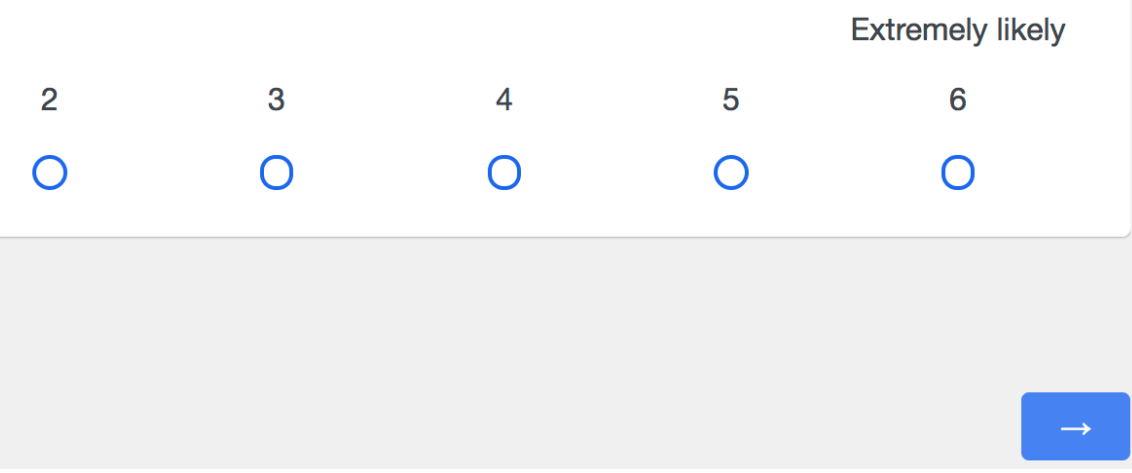

Figure 5. An example item from Experiment 2 as it appeared to participants in Qualtrics

3.2. PARTICIPANTS. Participants were recruited from Amazon Mechanical Turk. The analysis here includes data from 28 adult native speakers of American English, who reported normal hearing and normal (or corrected-to-normal) vision, and who had not previously participated in any related experiments. There were no explicit catch trials in Experiment 2; however, we used ratings of the inalienable nouns to filter out respondents who were not sufficiently attending to the task. We judged that an average person could be reasonably assumed to have at least one of all of the inalienable nouns (e.g. ear), and therefore we excluded any participant whose mean likelihood of possession rating for this condition was below 5 . We excluded 3 such participants.

3.3. RESULTS. We found no support for the theory that a noun's likelihood of possession influences its rate of bound variable interpretation. We used each participant's mean rating and standard deviation across all nouns to convert the raw ratings into z-scores. We then used Pearson's product moment correlation to assess the relationship between the mean z-scored rating for each noun and the proportion of bound variable interpretations which that noun produced in Experiment 1 . Ratings of possession likelihood did not correlate significantly with proportions of bound variable interpretation $(r=0.13, p=0.38$; see Figure 6$)$. 


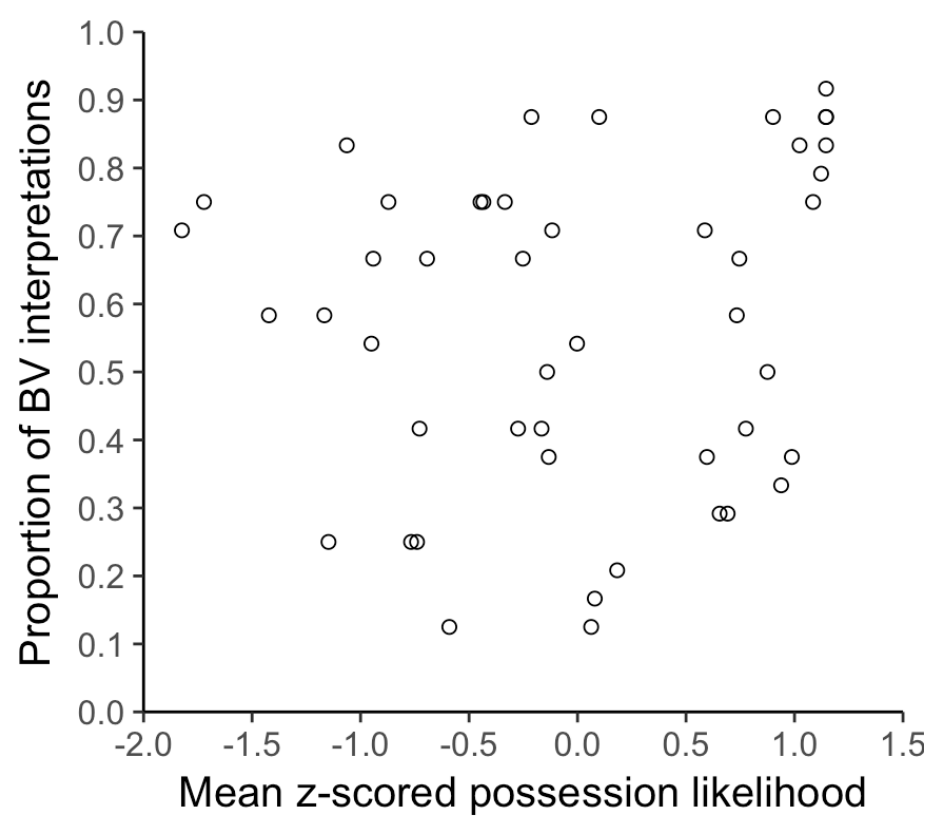

Figure 6. The relationship between each noun's possession likelihood and the proportion of bound variable interpretations which it produced in Experiment 1

3.4. DisCUSSION. The results of Experiment 2 demonstrate that people do possess high-level assumptions that certain nouns are more likely to be possessed by a character about whom they have no prior knowledge; however, we found no evidence that these assumptions influence the rate of bound variable interpretation for a sentence containing that possessed noun. These results argue against an account which prioritizes real-world plausibility as a predictor of bound variable bias. Instead, the results indirectly support our earlier hypothesis about the role of possession type.

4. General Discussion. The main aim of this study was to assess the effects of different possession relations on the comprehension of VP ellipsis structures which are ambiguous between bound variable and coreferential interpretations. Specifically, we sought to discriminate between a possessed noun's animacy and its alienability with regard to which of these factors has the larger influence on interpretational preference. We found that the possessed noun's animacy plays a significant role in ambiguity resolution and better explains the pattern of results observed in Experiment 1 and replications thereof. In Experiment 2, we ruled out the alternative hypothesis that real-world plausibility drives interpretational preference for these structures. $\mathrm{We}$ presented a simple model to account for the patterns observed in these experiments. In this model, possessed nouns differ in the extent to which their discourse representations depend on those of their possessors. This model posits that an independent discourse representation is more likely to be available for coreference when VP ellipsis is interpreted, but a dependent representation is more likely to result in a variable binding.

Development of the proposed model should provide abundant opportunity for future work. Currently this model is specified at the level of discourse representation and provides useful explanatory power in modeling offline comprehension; however, the eventual goal for such a model ought to be application to online processing. Future research of this sort should attempt to explain how these discourse representations are built up in real time and define the locus of the model's probabilistic behavior. To accomplish this goal, it will also likely be necessary to inte- 
grate the model with syntactic and semantic theories of VP ellipsis processing. Our proposal could additionally benefit from an explicit computational implementation, with the hope of informing future psycholinguistic inquiry into this topic.

Finally, one of the most intriguing results of Experiment 1 and the replication studies was the difference in bound variable bias between animate relational and kinship nouns. The most likely explanation given the current data seems to be that kin referents are simply more independent in discourse due to their cultural prominence and thus lead to more coreferential interpretations. In the future, it might be fruitful to look for additional continuous predictors relevant to nouns (similar to possession likelihood in Experiment 2) which could better explain the pattern of results. A useful continuous predictor or set of predictors could be an especially worthwhile addition to a computational implementation of the proposed model.

In sum, this study provides strong evidence that possession type influences the interpretation of ambiguous VP ellipsis and identifies animacy of the possessed noun (a variable previously untested in this domain) as an influential predictor of interpretational preference.

5. Appendix: Details of the replication studies in \$2.6. The two experiments reported above as replications of Experiment 1 were intended to explore an observation discussed at length in Oku (1998:99-112) - and previously reported in Fiengo \& May (1994), Kitagawa (1991), and Sag (1976) - firstly, that sentences similar to Example (1a) that involve gender mismatch between the possessive pronoun in the antecedent VP and its elided counterpart may be degraded under bound variable interpretation, and secondly, that the order of the mismatched genders interacts with the effect of this morphological mismatch.

a. Bill washed his car, and Susan did, too.

Bill $l_{i}$ washed hisi car, and Susan did $_{\text {[vp washed her }}$ car], too.

Bill $_{i}$ washed hisi car, and Susan $_{j}$ did [vp washed hisi car], too.

Bill $_{\mathrm{i}}$ washed hisk $\mathrm{k}_{\mathrm{k}}$ car, and $\mathrm{Susan}_{\mathrm{j}}$ did [vp washed his $\mathrm{k}_{\mathrm{k}}$ car], too.

(bound variable)

(coreference)

(coreference)

b. Mary washed her car, and John did, too.

?Mary $\mathrm{i}_{\mathrm{i}}$ washed her $\mathrm{i}$ car, and $\mathrm{John}_{\mathrm{j}}$ did [vP washed his $\mathrm{j}_{\mathrm{j}}$ car], too.

Maryi washed heri car, and $\mathrm{John}_{\mathrm{j}}$ did [vp washed her $\mathrm{i}_{\mathrm{i}}$ car], too.

(bound variable)

Maryi washed her ${ }_{k}$ car, and $\mathrm{John}_{\mathrm{j}}$ did [vp washed her $\mathrm{k}_{\mathrm{k}} \mathrm{car}$ ], too.

(coreference)

(coreference)

The essential generalization reported in previous work is that, with some degree of variation, most native English speakers are able to assign a bound variable interpretation to sentence (7a); however, many speakers find that the bound variable interpretation of (7b) is degraded. Previous theories of ellipsis resolution required additional mechanisms in order to deal with this observation; nevertheless, these judgments were never submitted to rigorous experimental verification, nor did previous work acknowledge the impact of the possessed noun's lexical semantic properties on the acceptability of bound variable interpretations. The replication experiments were primarily designed to verify previous judgments presented in the syntactic literature and secondly to examine the interaction of the gender mismatch effect and the pattern of results found in Experiment 1.

Methods and materials in these two experiments were substantially similar to those of Experiment 1 . Instead of manipulating possession type, conditions differed with respect to the gender of the two "characters" in each target item, as described below in Table 1. 


\begin{tabular}{lll} 
Match/Mismatch & Overt pronoun & Example Target Sentence \\
\hline Match & Masculine & Brad gweeshed his broom, and Marcus did, too. \\
Mismatch & Masculine & Brad gweeshed his broom, and Monica did, too. \\
Match & Feminine & Stephanie gweeshed her broom, and Monica did, too. \\
Mismatch & Feminine & Stephanie gweeshed her broom, and Marcus did, too. \\
\hline
\end{tabular}

Table 1. The four conditions in the two replication studies, with example target sentences

The two replication studies differed only with respect to the possession type of nouns in target sentences; one used ownership nouns, while the other used animate relational and kinship nouns (thus creating a $2 \times 2 \times 2$ design). As in Experiment 1, participants in the replication studies were recruited from Amazon Mechanical Turk and completed the experiments in Qualtrics.

In short, these two studies found no support for the judgments presented in prior syntactic literature, namely that bound variable interpretations of sentences like Example (7b) are dispreferred. In fact, the gender manipulation in these experiments did not significantly affect participants' preferences, and all gender combinations received similar rates of bound variable interpretation with possession type held constant. Nevertheless, we did observe differences across possession types, and these patterns mirrored those in Experiment 1. Accordingly, the results reported in $\$ 2.6$ collapse across the non-differing gender conditions. With regard to the judgments reported in Oku (1998) and elsewhere, we adopt the provisional conclusion that speakers of American English - in its current state, at least - do not discriminate between bound variable interpretations of Examples (7a) and (7b).

\section{References}

Bates, Douglas, Martin Mächler, Ben Bolker, \& Steve Walker. 2015. Fitting linear mixed-effects models using lme4. Journal of Statistical Software 67(1). 1-48. https://doi.org/10.18637/jss.v067.i01.

Dahl, Östen \& Kari Fraurud. 1996. Animacy in grammar and discourse. In Thorstein Fretheim and Jeanette K. Gundel (eds.), Reference and Referent Accessibility. Pragmatics \& Beyond New Series, 47-64. Amsterdam: John Benjamins. https://doi.org/10.1075/pbns.38.04dah.

Fiengo, Robert, \& Robert May. 1994. Indices and identity. Cambridge, MA: MIT Press.

Foley, Claire, Zelmira Núñez del Prado, Isabella Barbier, \& Barbara Lust. 2003. Knowledge of variable binding in VP-ellipsis: Language acquisition research and theory converge. Syntax 6(1), 52-83. https://doi.org/10.1111/1467-9612.00056.

Frazier, Lyn, \& Charles Clifton, Jr. 2000. On bound variable interpretations: The LF-only hypothesis. Journal of Psycholinguistic Research 29(2), 125-140.

http://dx.doi.org/10.1023/A:1005136826534.

Grodzinsky, Yosef, Kenneth Wexler, Yu-Chin Chien, Susan Marakovitz, \& Julie Solomon. 1993. The breakdown of binding relations. Brain and Language 45(3), 396-422.

https://doi.org/10.1006/brln.1993.1052.

Guo, Fangfang, Claire Foley, Yu-Chin Chien, Chi-Pang Chiang, \& Barbara Lust. 1996. Operator-variable binding in the initial state: A cross-linguistic study of VP ellipsis structures in Chinese and English. Cahiers de linguistique - Asie orientale 25(1), 3-34. https://doi.org/10.3406/clao.1996.1490.

Haspelmath, Martin. 2017. Explaining alienability contrasts in adpossessive constructions: Predictability vs. iconicity. Zeitschrift für Sprachwissenschaft 36(2), 193-231. https://doi.org/10.1515/zfs-2017-0009. 
Heim, Irene. 1982. The semantics of definite and indefinite noun phrases. Amherst, MA: University of Massachusetts dissertation.

Hollmann, Willem \& Anna Siewierska. 2007. A construction grammar account of possessive constructions in Lancashire dialect: Some advantages and challenges. English Language and Linguistics 11(2), 407-424. https://doi.org/10.1017/S1360674307002304 Kitagawa,

Yoshihisa. 1991. Copying identity. Natural Language \& Linguistic Theory 9(3). 497536. https://doi.org/10.1007/BF00135356.

Koornneef, Arnout W., Sergey Avrutin, Frank Wijnen, \& Eric Reuland. 2011. Tracking the preference for bound-variable dependencies in ambiguous ellipses and only-structures. In Jeffrey T. Runner (ed.), Experiments at the interfaces. Syntax and semantics (37), 67-100. New York: Emerald Publishing. https://doi.org/.1163/9781780523750 004.

Oku, Satoshi. 1998. A theory of selection and reconstruction in the minimalist perspective. Storrs, CT: University of Connecticut dissertation.

Ong, Matthew \& Adrian Brasoveanu. 2014. Strict and sloppy reflexives in VP ellipsis. In Christopher Piñón (ed.), Empirical Issues in Syntax and Semantics 10, 251-268.

http://www.cssp.cnrs.fr/eiss10/eiss10 ong-and-brasoveanu.pdf.

R Core Team. 2016. R: A language and environment for statistical computing. R Foundation for Statistical Computing. Vienna, Austria. https://www.R-project.org/.

Reuland, Eric. 2001. Primitives of binding. Linguistic Inquiry 32(3), 439-492. https://doi.org/10.1162/002438901750372522.

Sag, Ivan A. 1976. Deletion and logical form. Cambridge, MA: MIT dissertation. http://hdl.handle.net/1721.1/16401

Shapiro, Lewis P. \& Arild Hestvik. 1995. On-line comprehension of VP-ellipsis: Syntactic reconstruction and semantic influence. Journal of Psycholinguistic Research 24(6), 517-532. https://doi.org/10.1007/BF02143165.

Shapiro, Lewis P., Arild Hestvik, Lesli Lesan, \& A. Rachel Garcia. 2003. Charting the timecourse of VP-ellipsis sentence comprehension: Evidence for an initial and independent structural analysis. Journal of Memory and Language 49(1), 1-19. https://doi.org/10.1016/S0749-596X(03)00026-3.

Vasić, Nada, Sergey Avrutin, \& Esther Ruigendijk. 2006. Interpretation of pronouns in VPellipsis constructions in Dutch Broca's and Wernicke's aphasia. Brain and Language 96(2). 191-206. https://doi.org/10.1016/j.bandl.2005.04.003. 